\title{
EIGENSTRUCTURE TECHNIQUES FOR FAULT DETECTION AND ISOLATION IN AEROSPACE AND AUTOMOTIVE SYSTEMS
}

\author{
Adilson de Jesus Teixeira \\ IAE - Instituto de Aeronáutica e Espaço \\ Marcelo Lopes de Oliveira e Souza \\ INPE - Instituto Nacional de Pesquisas Espaciais \\ Álvaro Prieto Oliva \\ EMBRAER - Empresa Brasileira de Aeronáutica
}

Copyright (C) 2004 Society of Automotive Engineers, Inc.

\begin{abstract}
Eigenstructure techniques allow to detect and isolate faulty components in a dynamic process, such as sensor biases, actuator malfunctions, changes in dynamic parameters due to leaks and deterioration. Fault detection is the first step to achieve fault tolerance, but for this the redundancy has to be included in the system. This redundancy can be either by hardware or by software. In situations in which it is not possible to use hardware redundancy only the software redundancy can be used.
\end{abstract}

Therefore using eigenstructure techniques, for the fault detection and isolation, the tests can be done through the angle between the residue vector direction and the fault direction vector. By this way, we can reduce false alarm and the alarm loss rates due to the noise and changes in system parameters.

\section{INTRODUCTION}

In several cases, the fault in a component can be catastrophic if the control does not have any redundancy degree, physical or analytical. Therefore, it is important to implement a control system with redundancy and capacity to identify faults in the components as fast as possible, such a way, that it can reconfigure the use of the remaining components or even the control law with faults to an alternative control law.

A detection and isolation technique is an algorithm to detect and isolate (locate) faulty components in a dynamic process, such as sensor biases, actuator malfunctions, leaks and equipment deterioration. Fault detection is the first step to achieve fault tolerance, but for this the redundancy has to be included in the system. The redundancy can be either by hardware (physical redundancy) or by software (analytical redundancy). Hardware redundancy, e.g., an extra sensor or extra actuator, can produce several problems associated with: cost, space, weight and complexity of the system. Besides, it has been observed that redundant components tend to have similar functioning expectancies; so the event that causes one component to fail probably could cause its redundant components to fail soon. There are even situations in which it is not possible to use hardware redundancy; in this case, only the analytical redundancy approach can be used. Analytical redundancy uses a mathematical model of the system, so this approach only needs some computational resources of the system.

Eigenstructure techniques make use of analytical redundancy approach based on the system mathematical model. Through these techniques we can see that a single fault in one component of the system has one specific direction (signature) independent of its amplitude changes. So, the fault detection and isolation is done through the test of the vector directions instead of their amplitudes. By this way, we can reduce false alarm and the alarm loss rates due to the sensor noises and changes in system parameters. Another advantage is the implementation feasibility of the analytical redundancy since nowadays the control systems are design with an appreciable process capacity. The main stages of these techniques are: 1) observer dynamic definition; 2) residual generations using the mathematical models; 3) signature fault generation; and 4) signature tests 
for detection and isolation.

This paper presents some eigenstructure techniques for fault detection and isolation that can be applied in aerospace or automotive system. For results evaluation, it is presented some examples using an aerospace vehicle model for inclusion of instrument fault detection (IFD).

\section{BASIC CONCEPTS}

FAULT - Fault can be defined as a malfunction of any component of a system, causing since a loss of performance up to a total stop of its functions. According to [1] the faults can be divided in:

- Abrupt Fault: fault that suddenly occurs and persists in a component.

- Incipient Fault: fault that develops slowly at a component.

The early detection of an incipient fault can help to avoid a total breakdown of the system or even catastrophes that could result in loss of significant amount of material or serious personal injury. So, it is desired to have a fault tolerant system, that is, a system that can continue to do its task, even when there are hardware faults or software errors. But the implementation of such system is not easy to do.

We will use the word fault because strictly speaking, the term failure suggests complete breakdown, while fault may connote something tolerable.

According to the terminology used in [2], the fault detection and diagnosis consist of the following tasks:

- Fault Detection: detection that something is wrong in the system. Special emphasis is laid upon incipient faults rather than abrupt faults because incipient faults are harder to detect.

- Fault Isolation: determination of the fault origin.

- Fault Identification: determination of the gravity of the fault.

Other relevant aspects, in fault detection and isolation, are the problems relative to the false alarm and alarm loss. False Alarm is the indication of the occurrence of a fault when the system is operating in its normal condition. Alarm Loss, on the other hand, is the indication that the system is operating normally, when it is in a faulty condition. The decision threshold between the fault state and normal state of operation should be chosen in such a way to minimize these two wrong and conflicting indications.

A wide class of fault detection and isolation methods makes explicit use of the system mathematical model, such as the model-based methods, which are based on the idea of the analytical redundancy [3]. In contrast with the physical redundancy, where measurements of different sensors are compared, in the analytical redundancy the measurements supplied by a sensor are compared with the respective variable value obtained through the mathematical model. Such value is obtained through calculations that use current and/or previous measurements of another variable and the mathematical model that describes their relationships. The idea can still be extended only for the comparison of the values generated analytically, each of them being obtained through different calculations. In both cases, the resultant differences are called of residues.

The residues have zero value in ideal situations but in practice this rarely happens. The deviations of this value are a combined result of the noise, parameters change and/or faults. If the deviations are negligible, the residues for eigenstructure technique can be analyzed directly for the direction of the fault. In the presence of significant deviation it is necessary to do a statistical analysis. In both cases the directions are generated, indicating which direction can be considered for each fault. Such directions are called fault directions.

RESIDUES - For approaching studies on residues where the deviations are negligible, according to [4], some definitions are necessary: A Residual (Parity Vector) $\boldsymbol{r}(t)$ is a scalar or vector that has null or very small values, in the faults absence, and has values different from zero when a fault occurs. The residual is, therefore, a vector in the parity space. This definition implies that a residual $\boldsymbol{r}(t)$ has to be independent of, or at least, insensitive to system states and unmeasured disturbances.

In case of linear systems, a general structure of a linear residual generator can be described as in the Figure 1. The transfer function from the fault matrix $\boldsymbol{F}(t)$ to the residual matrix $\boldsymbol{R}(t)$ is given by:

$$
\boldsymbol{R}(s)=\boldsymbol{H}_{y}(s) \boldsymbol{G}_{f}(s) \boldsymbol{F}(s)
$$

The condition, for the system to be able to detect a fault in the residual, is based on the detectability definition. Detectability is the capability to detect the $i^{\text {th }}$ fault that corresponds to the $i^{\text {th }}$ column of the response matrix $\boldsymbol{R}(s)$ that is different from zero.

This condition, however, it is not enough in some practical situations. Assume that we have two residual generators as presented in the Figure 1. And in occurrence of a fault the residuals behave as in Figure 2. Here we see that we have a fundamentally different behavior between $\boldsymbol{r}_{1}(t)$ and $\boldsymbol{r}_{2}(t)$, because $\boldsymbol{r}_{1}(t)$ only reflects transitions on the faulty signal while $\boldsymbol{r}_{2}(t)$ has approximately the same shape of the faulty signal. Thus, $\boldsymbol{r}_{1}(t)$ can not be used in a reliable IFD application even though it is clear that $\boldsymbol{R}_{l}(s) \neq 0$.

The difference between the two residuals in this example is the values of $\boldsymbol{R}(s=0)$. Clearly we can see that the residual 1 has $\boldsymbol{R}_{l}(s=0)=0$ while the residual 2 has 
$\boldsymbol{R}_{2}(s=0) \neq 0$. This leads to the definition of strong detectability. The $i^{\text {th }}$ fault is said to have strong detectability if and only if:

$$
\boldsymbol{R}_{i}(s=0) \neq 0
$$

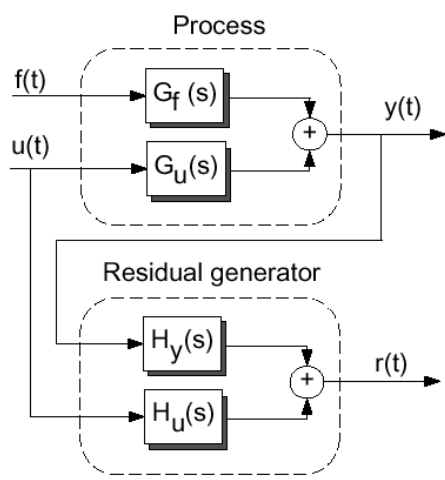

Figure 1-General structure of a linear residual generator.
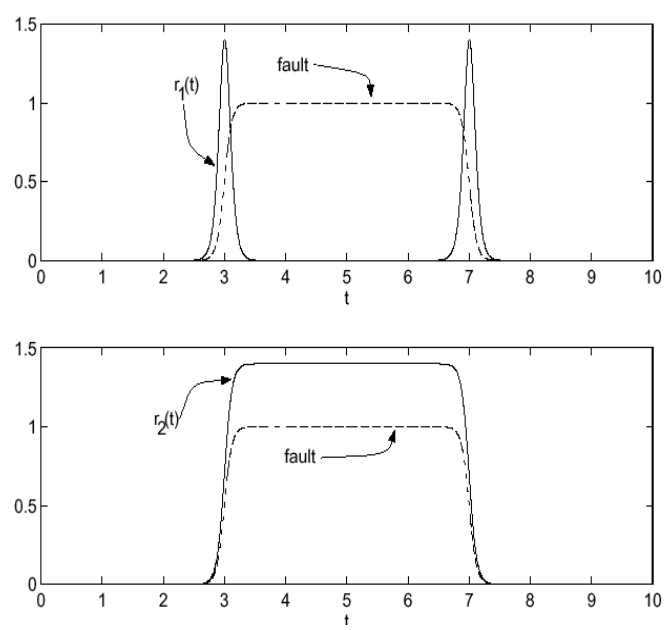

Figure 2 - Residuals examples.

EIGENSTRUCTURE - The eigenstructure approach is a closed-loop observer based method aiming to make the residuals, not the estimated state, insensitive to disturbances or to have a desired direction. It can easily be extended to residual generator to facilitate fault isolation. The eigenstructure of a matrix $\boldsymbol{A}$ is the set $\left\{\lambda_{i}, \boldsymbol{v}_{i}\right\}_{i=1, \ldots, \mathrm{n}}$, where $\lambda_{i}$ are the eigenvalues an $\boldsymbol{v}_{i}$ are the eigenvectors.

FIXED DIRECTIONS RESIDUALS - According to [4], this conception is the base of the fault detection filter where the residual vector get a specific direction depending on the fault that is acting upon the system. Figure 3 gives a geometrical representation of this type of residual when a sensor fault has occurred. The most probable fault can be determined by finding the fault vector is approximately parallel to the residual.

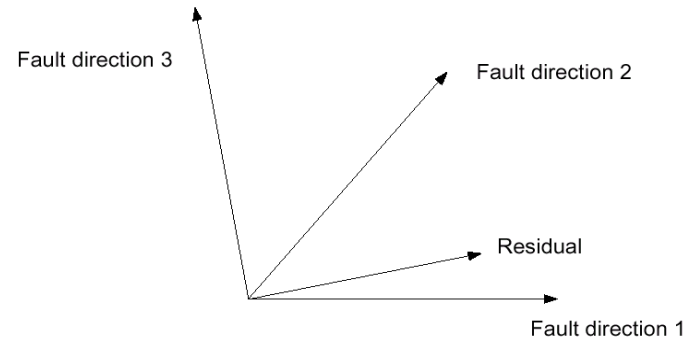

Figure 3 - Fixed direction residual.

ROBUSTNESS REQUIREMENTS - According to [4], a problem to be considered is that unmeasured signals and modeling error are always present in the system. This makes it hard to keep the false alarm rate at an appropriate level. If it is known how the uncertainties influence the process, these uncertainties are denominated structured uncertainties. This information can be used to reduce or even eliminate their influence on the residuals. If it is not known how disturbances act upon the system, there is a little that can be done to decouple these influences. Actually we have not designed any robustness, the best than we can do is to maximize the sensibility to the faults and to minimize the sensibility to the disturbances over all operation points.

However it is possible to increase the robustness in the fault evaluation stage by using adaptive threshold levels or statistical decoupling in the step of decision threshold selection. This is called passive robustness. It is not likely that one method can solve the entire robustness problem; a likely solution is one where disturbance decoupling is used side by side with adaptive thresholds.

\section{MODEL BASED STRUCTURE}

In this paper, a model of a linear system, with time invariant parameters, represented in continuous time state space is given by:

$$
\begin{aligned}
& \dot{\boldsymbol{x}}(t)=\boldsymbol{A} \boldsymbol{x}(t)+\boldsymbol{B} \boldsymbol{u}(t) \\
& \boldsymbol{y}(t)=C \boldsymbol{x}(t)+\boldsymbol{D} \boldsymbol{u}(t)
\end{aligned}
$$

where $\boldsymbol{A}$ is a $n \times n$ system dynamic matrix; $\boldsymbol{B}$ is a $n \times r$ control matrix; $\boldsymbol{C}$ is a $p \times n$ output matrix; $\boldsymbol{D}$ is a $p \times r$ direct feedthrough matrix; $\boldsymbol{x}(t)$ is a $n \times 1$ state vector; $\boldsymbol{u}(t)$ is a $r \times 1$ control vector; and $\boldsymbol{y}(t)$ is a $p \times 1$ output vector.

We considered three types of general of faults:

1. Sensor Fault: modeled here as an additive fault of the plant output signal; 
2. Actuator Fault: modeled as an additive fault of the input signal in the system dynamics, and

3. Component Fault: modeled as a multiplicative fault, i. e., any distribution matrix that is added to the system dynamics matrix.

We used the following fault cases for studies [5]:

- Zero: when the sensor (actuator or component) begins to supply only the zero value, that is, the sensor has an abrupt variation to the value zero;

- Maximum Value: when the sensor begins to supply only the maximum value in module, that is, the sensor has an abrupt variation to its maximum or minimum value;

- Constant: when the sensor begins to supply the last measurement made before the fault occurs;

- Offset Drift: when the value of the offset alters the measurement in function of time, and

- Scale Factor Drift: when the scale factor of the sensor alters the measurement in function of time.

There are also uncertainties about the model and unmeasured inputs in the process. If these uncertainties are structured, that is, it is known how they enter in the system dynamics; this information can be incorporated into the model.

In case of linear model with structured uncertainties, based on [4], the complete model can be represented by:

$$
\begin{aligned}
& \dot{\boldsymbol{x}}(t)=\boldsymbol{A} \boldsymbol{x}(t)+\boldsymbol{B}\left[\boldsymbol{u}(t)+\boldsymbol{f}_{a}(t)\right]+\boldsymbol{H} \boldsymbol{f}_{\boldsymbol{c}}(t)+\boldsymbol{E} \boldsymbol{d}(t) \\
& \boldsymbol{y}(t)=\boldsymbol{C} \boldsymbol{x}(t)+\boldsymbol{D} u(t)+\boldsymbol{f}_{\boldsymbol{s}}(t)
\end{aligned}
$$

where $f_{d}(t)$ denotes actuator faults, $f_{c}(t)$ is for component faults, $\boldsymbol{f}_{s}$ is for sensor faults, $\boldsymbol{d}(t)$ is for disturbances acting upon the system, $\boldsymbol{H}$ is the distribution matrix for components fault and $\boldsymbol{E}$ is the distribution matrix for disturbances acting upon the system.

RESIDUAL GENERATOR - The residual generator is based on a straightforward state estimator observer [7] represented by:

$$
\begin{aligned}
& \dot{\hat{\boldsymbol{x}}}(t)=\boldsymbol{A} \hat{\boldsymbol{x}}(t)+\boldsymbol{B} \boldsymbol{u}(t)+\boldsymbol{L}[\boldsymbol{y}(t)-\hat{\boldsymbol{y}}(t)] \\
& \hat{\boldsymbol{y}}(t)=\boldsymbol{C} \hat{\boldsymbol{x}}(t)+\boldsymbol{D} \boldsymbol{u}(t)
\end{aligned}
$$

To facilitate the description of the equations, the terms that indicate functions of $t$ will be omitted; and bold letters will identify the matrices and vectors.

Considering $\boldsymbol{e}=\boldsymbol{x}-\hat{\boldsymbol{x}}$ we get the estimation error dynamics as:

$$
\dot{\boldsymbol{e}}=\dot{\boldsymbol{x}}-\dot{\hat{\boldsymbol{x}}}=(\boldsymbol{A}-\boldsymbol{L C}) \boldsymbol{e}+\boldsymbol{B \boldsymbol { f } _ { a }}+\boldsymbol{H} \boldsymbol{f}_{c}+\boldsymbol{E d}-\boldsymbol{L} \boldsymbol{f}_{s}
$$

and the residual is formed by:

$$
\boldsymbol{r}=\boldsymbol{y}-\hat{\boldsymbol{y}}=\boldsymbol{C x}+\mathrm{D} \boldsymbol{u}+\boldsymbol{f}_{s}-\boldsymbol{C} \hat{\boldsymbol{x}}-\mathrm{Du}
$$

$$
\boldsymbol{r}=\boldsymbol{C e}+\boldsymbol{f}_{s}
$$

Robust Residual Generator- The robust residual generator is formed by premultiplying the output estimation error to achieve insensitivity:

$$
\boldsymbol{r}_{w}=\boldsymbol{W}(\boldsymbol{y}-\hat{\boldsymbol{y}})=\boldsymbol{W C e}+\boldsymbol{W} \boldsymbol{f}_{s}
$$
by:

This residual in the frequency domain is represented

$$
\boldsymbol{R}_{w}(s)=\boldsymbol{W C}\left(s \boldsymbol{I}-\boldsymbol{A}_{o}\right)^{-1}\left(\boldsymbol{B} \boldsymbol{f}_{a}+\boldsymbol{H} \boldsymbol{f}_{c}+\boldsymbol{E} \boldsymbol{d}-\boldsymbol{L} \boldsymbol{f}_{s}\right)+\boldsymbol{W} \boldsymbol{f}_{s}
$$

where $\boldsymbol{A}_{o}=\boldsymbol{A}-\boldsymbol{L C}$

The disturbance decoupling condition can now easily be seen as:

$$
\boldsymbol{G}(\mathrm{s})=\boldsymbol{W C}\left(s \boldsymbol{I}-\boldsymbol{A}_{o}\right)^{-1} \boldsymbol{E}=0
$$

It is easy to see that the actuator faults can also be thought as unmeasured disturbances that enter into the system dynamics through the matrix $\boldsymbol{B}$. To achieve actuator fault decoupling we can enlarge the matrix $\boldsymbol{E}$ with the columns of matrix $\boldsymbol{B}$ that contain the fault that we need to decouple. Components faults can be considered of the same way. For sensor faults we have another approach: since the observer is driven by sensor measurements, we only need to skip the feedback from the sensor whose fault we want to decouple. The problem is now how to find the matrices $\boldsymbol{W}$ and $\boldsymbol{L}$ that fulfills the equation above.

Before we proceed and describe a method to find $\boldsymbol{W}$ and $\boldsymbol{L}$ to achieve disturbance decoupling, we need some additional mathematical tools regarding eigenstructure, i. e., eigenvalues and eigenvectors. The eigenvalues and eigenvectors of the closed-loop observer matrix can be chosen to provide disturbance decoupling or a desired direction. In the continuous-time case an asymptotic form of robust fault monitor can be designed whilst in the discretetime case the dead-beat observer can be designed directly through assigning $p$ zero-value eigenvalues corresponding to $p$ sensor channels. With this information we can determine what will be the direction of the fault in order to design the detection filter.

Lemma 1 - According to [4] if a matrix $\boldsymbol{A}$ has eigenvalues $\left\{\lambda_{i}\right\}_{i=1, \ldots, \mathrm{n}}$ then $\boldsymbol{A}^{T}$ has the same set of eigenvalues. This is equivalent to say that the left and right eigenvectors of a matrix have the same set of eigenvalues.

Lemma 2 - According to [4] assume $\boldsymbol{A}$ has right eigenvectors $\left\{\boldsymbol{v}_{i}\right\}_{i=1, \ldots, \mathrm{n}}$ and left eigenvectors $\left\{\boldsymbol{I}_{i}\right\}_{i=1, \ldots, \mathrm{n},}$, corresponding to the eigenvalues $\left\{\lambda_{i}\right\}_{i=1, \ldots, \mathrm{n}}$. As noted in Lemma 1, left and right eigenvectors have the same set of eigenvalues. Then a given left eigenvector $\boldsymbol{l}_{i}$ is always orthogonal to the right eigenvector $\boldsymbol{v}_{j}$, i. e.: 


$$
\boldsymbol{l}_{i}^{T} \boldsymbol{v}_{j} \begin{cases}=0 & , \text { if } \lambda_{i} \neq \lambda_{j} \\ \neq 0 & \text {,if } \lambda_{i}=\lambda_{j}\end{cases}
$$

Proof - By definition we have:

$$
\begin{aligned}
& \lambda_{i} \boldsymbol{v}_{i}=\boldsymbol{A} \boldsymbol{v}_{i} \\
& \lambda_{i} \boldsymbol{l}_{i}^{T}=\boldsymbol{l}_{i}^{T} \boldsymbol{A}
\end{aligned}
$$

By post multiplying eq. (13) by vector $\boldsymbol{v}_{\mathrm{j}}$ we get:

$$
\begin{gathered}
\lambda_{i} \boldsymbol{l}_{i}^{T} \boldsymbol{v}_{j}=\boldsymbol{l}_{i}^{T} \boldsymbol{A} \boldsymbol{v}_{j}=\lambda_{j} \boldsymbol{l}_{i}^{T} \boldsymbol{v}_{j} \\
\left(\lambda_{i}-\lambda_{j}\right) \boldsymbol{l}_{i}^{T} \boldsymbol{v}_{j}=0
\end{gathered}
$$

which ends the proof.

The residue dynamics $\left(s \boldsymbol{I}-\boldsymbol{A}_{o}\right)^{-1}$, originated from the state estimator observer dynamics, can be expanded in several ways, resulting in different design methods for the residual generators.

Lemma 3 - According to [4], the resolvent $(s \boldsymbol{I}-\boldsymbol{A})^{-1}$ can be expanded as:

$$
(s \boldsymbol{I}-\boldsymbol{A})^{-1}=\frac{\boldsymbol{I}}{s}+\frac{\boldsymbol{A}}{s^{2}}+\cdots+\frac{\boldsymbol{A}^{m}}{s^{m+1}}+\cdots
$$

The proof can be found in [4].

Applying Lemma 3 to eq. (10), the decoupling condition becomes:

$$
\begin{aligned}
& \boldsymbol{W} \boldsymbol{C} \boldsymbol{A}^{i} \boldsymbol{E}=0 ; \quad i=1 \ldots n-1 \\
& \boldsymbol{W} \boldsymbol{C} \boldsymbol{E}=0
\end{aligned}
$$

Note that $i$ only goes up to $n-1$, as a direct consequence of Cayley-Hamilton's theorem as showed below.

Theorem 1- A square matrix satisfies its own characteristic equation:

$\operatorname{det}(\lambda \boldsymbol{I}-\boldsymbol{A})=\boldsymbol{A}^{n}+a_{1} \boldsymbol{A}^{n-1}+\ldots+a_{n-1} \boldsymbol{A}+a_{n}=0$

Proof: If we right multiply the characteristic equation by its respective eigenvector we get:

$$
\left(\lambda_{i}^{n}+a_{1} \lambda_{i}^{n-1}+\ldots+a_{n-1} \lambda_{i}+a_{n}\right) \mathbf{v}_{\mathrm{i}}=0
$$

From Lemma 2 we have: $\boldsymbol{A} \boldsymbol{v}_{i}=\lambda_{i} \boldsymbol{v}_{i}$, so

$$
\lambda_{i}^{2} \boldsymbol{v}_{i}=\lambda_{i} \lambda_{i} \boldsymbol{v}_{i}=\lambda_{i} \boldsymbol{A} \boldsymbol{v}_{i}=\boldsymbol{A} \lambda_{i} \boldsymbol{v}_{i}=\boldsymbol{A}^{2} \boldsymbol{v}_{i}
$$

Using the same considerations for all terms we get:

$$
\begin{array}{r}
\boldsymbol{A}^{n}+a_{1} \boldsymbol{A}^{n-1}+\ldots+a_{n-1} \boldsymbol{A}+a_{n}=0 \\
\boldsymbol{A}^{n}=-a_{1} \boldsymbol{A}^{n-1}-\ldots-a_{n-1} \boldsymbol{A}-a_{n}
\end{array}
$$

Therefore we conclude that any matrix of order $n$ or higher can be written as a linear combination of $\left\{\boldsymbol{A}^{i}\right\}_{i=1 \ldots n-1}$, which ends the proof.
So it is possible to find $\boldsymbol{L}$ and $\boldsymbol{W}$ in such a way that the rows of $\boldsymbol{W C}$ are left eigenvectors of $\boldsymbol{A}_{o}$ and, at the same time, keeps $\boldsymbol{W C} \boldsymbol{E}=0$. Then, decoupling the disturbances from faults according to eqs. (15) and (16), may be achieved.

Lemma 4 - According to [4], the resolvent $(s \boldsymbol{I}-\boldsymbol{A})^{-1}$ can also be expanded on its eigenstructure as:

$$
(s \boldsymbol{I}-\boldsymbol{A})^{-1}=\sum_{i=1}^{n} \frac{\boldsymbol{v}_{\boldsymbol{i}} \boldsymbol{l}_{i}^{T}}{\mathrm{~s}-\lambda_{\mathrm{i}}}
$$

where $v_{i}$ and $\boldsymbol{l}_{i}$ are the right and left eigenvectors corresponding to the eigenvalues $\lambda_{i} . \boldsymbol{v}_{\mathrm{i}}$ and $\boldsymbol{l}_{i}$ must be scaled so that $\boldsymbol{v}_{i}^{T} \boldsymbol{l}_{i}=1$; and $n$ is the order of matrix $\boldsymbol{A}$. The proof can be found in [4].

Theorem $2-$ According to [4], if $\boldsymbol{W C E}=0$ and all $p$ rows of matrix $\boldsymbol{W C}$ are left eigenvectors of $\boldsymbol{A}_{o}$ then eq. (15) is satisfied, where $p$ is the dimension of the residual.

Proof. According to [4], the rows of matrix $\boldsymbol{W C}$ are left eigenvectors of $\boldsymbol{A}_{o}$, i. e.:

$$
\boldsymbol{W C}=\left[\begin{array}{llll}
\boldsymbol{l}_{1}{ }^{T} & \boldsymbol{l}_{2}{ }^{T} & \cdots & \boldsymbol{l}_{p}{ }^{T}
\end{array}\right]^{T}
$$

The decoupling condition given by equation (15) can be represented by:

$$
\boldsymbol{W C} \sum_{i=1}^{n} \frac{\boldsymbol{v}_{i} \boldsymbol{l}_{i}^{T}}{s-\rho_{i}} \boldsymbol{E}=0
$$

According to Lemma 2, and since all rows of $\boldsymbol{W C}$ are the left eigenvectors, $\boldsymbol{l}_{i}$ for $i=1 \ldots p$, we get $\boldsymbol{W C} \boldsymbol{v}_{i}=0$ for $i=p+1 \ldots n$. The decoupling condition above can be written as:

$$
\boldsymbol{W C} \sum_{i=1}^{p} \frac{\boldsymbol{v}_{\boldsymbol{i}} \boldsymbol{l}_{i}^{T}}{\mathrm{~s}-\rho_{\mathrm{i}}} \boldsymbol{E}=\boldsymbol{0}
$$

But as it was assumed that $\boldsymbol{W C E}=0$, i. e., $\boldsymbol{l}_{i}^{T} \boldsymbol{E}=0$, $i=1, \ldots, p$, which yields

$$
\boldsymbol{W C}\left(S \boldsymbol{I}-\boldsymbol{A}_{\diamond}\right)^{-1} \boldsymbol{E}=0
$$

ending the proof.

Eigenstructure Assignment Details - Patton [1] shows that the observer feedback eigenstructure assignment problem can be handled through a transformation into the dual control form. As we have many methods available to compute accurately the feedback gains for either the full state feedback or the output feedback control problems we can extend theses techniques to the observer design case. The dual control problem can be solved by substituting $\boldsymbol{B}$ for $\boldsymbol{C}^{T}, \boldsymbol{A}$ for $\boldsymbol{A}^{T}$ and $\boldsymbol{K}$ for $\boldsymbol{L}^{T}$ in the methods for compute the feedback gains. So, by this consideration we will present the project in the dual control form. Based in Moore [8] we have the necessary and sufficient conditions for a general 
state feedback matrix to exist, which satisfies the standard control problem. These equations in the dual control form is given by:

$$
\left(\boldsymbol{A}^{T}-C^{T} \boldsymbol{L}^{T}\right) \boldsymbol{l}_{i}=\rho_{i} \boldsymbol{l}_{i} ; i=1, \ldots, n
$$

The necessary and sufficient conditions to find a real observer gain matrix $\boldsymbol{L}$ satisfying eq (19) are:

1. The $\boldsymbol{l}_{i} \in \mathrm{C}^{\mathrm{n}}$ are linearly independent vectors

2. $\boldsymbol{l}_{i}=\boldsymbol{l}_{j}^{*}$, whenever $\rho_{i}=\rho_{j}{ }^{*}$

Patton [1] shows that the subspace where the eigenvectors can be assigned are completely determined by the eigenvalue (and the system). To derive a base for the subspace we first need to make some definitions, with each eigenvalue $\rho_{i}$ associated to two matrices: $\boldsymbol{Q}\left(\rho_{i}\right)$ and $\boldsymbol{S}\left(\rho_{i}\right)$ :

$$
\begin{aligned}
& \boldsymbol{Q}\left(\rho_{i}\right)=\left(\rho_{i} \boldsymbol{I}-\boldsymbol{A}^{T} \mid \boldsymbol{C}^{T}\right) \in \mathfrak{R}^{n x(n+p)} \\
& \boldsymbol{S}\left(\rho_{i}\right)=\left[\begin{array}{ll}
\boldsymbol{P}\left(\rho_{i}\right) & \boldsymbol{T}\left(\rho_{i}\right)
\end{array}\right]^{T} \in \boldsymbol{R}^{(n+p) x p}
\end{aligned}
$$

where the columns of $\boldsymbol{S}\left(\rho_{i}\right)$ forms a basis for the null space of $\boldsymbol{Q}\left(\rho_{i}\right)$, i. e.:

$$
\boldsymbol{Q}\left(\rho_{i}\right) \boldsymbol{S}\left(\rho_{i}\right)=0
$$

If we post multiply eq. (20) by one vector $\boldsymbol{e}_{i}$ of length $p$ and perform the matrix multiplication we get:

$$
\begin{aligned}
\boldsymbol{Q}\left(\rho_{i}\right) \boldsymbol{S}\left(\rho_{i}\right) \boldsymbol{e}_{i} & =\left(\rho_{i} \boldsymbol{I}-\boldsymbol{A}^{T} \mid \boldsymbol{C}^{T}\right)\left[\boldsymbol{P}\left(\rho_{i}\right) \quad \boldsymbol{T}\left(\rho_{i}\right)\right]^{T} \boldsymbol{e}_{i} \\
& =\left(\rho_{i} \boldsymbol{I}-\boldsymbol{A}^{T}\right) \boldsymbol{P}\left(\rho_{i}\right) \boldsymbol{e}_{i}+\boldsymbol{C}^{T} \boldsymbol{T}\left(\rho_{i}\right) \boldsymbol{e}_{i}=0
\end{aligned}
$$

We can rewrite eq. (19) as:

$$
\left(\rho_{i} \boldsymbol{I}-\boldsymbol{A}^{T}\right) \boldsymbol{l}_{i}-C^{T} \boldsymbol{L}^{T} \boldsymbol{l}_{i}=0
$$

Comparing eqs (21) and (22) we get:

$$
\begin{aligned}
& \boldsymbol{l}_{i}=\boldsymbol{P}\left(\rho_{i}\right) \boldsymbol{e}_{i} \\
& \boldsymbol{L}^{T} \boldsymbol{l}_{i}=\boldsymbol{T}\left(\rho_{i}\right) \boldsymbol{e}_{i}=\boldsymbol{z}_{i}
\end{aligned}
$$

We can see in eq (23) that $\boldsymbol{l}_{i}$ is spanned by the columns in $\boldsymbol{P}\left(\rho_{i}\right)$ and through eq (24) we can calculate the observer gain matrix $\boldsymbol{L}$.

The problems now are how to find the $\boldsymbol{S}\left(\rho_{i}\right)$, given $\boldsymbol{Q}\left(\rho_{i}\right)$, and how to find $\boldsymbol{e}_{i}$ given the desired eigenvectors. As noted earlier $\boldsymbol{S}\left(\rho_{i}\right)$ is a basis for the null space of $\boldsymbol{Q}\left(\rho_{i}\right)$. There are several ways to find a null space basis for a matrix, several of them use invertion of matrices that may lead to numerical problems. Patton [1] shows a procedure based on Singular Value Decomposition (SVD). Applying a SVD to $\boldsymbol{Q}\left(\rho_{i}\right)$ we get:

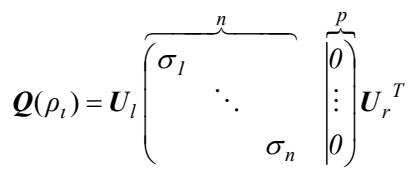

As $\boldsymbol{U}_{r}$ is an orthogonal matrix, the last $p$ columns of the product $\boldsymbol{Q}\left(\rho_{i}\right) \boldsymbol{U}_{r}$ will be $\mathbf{0}$. It should be noted that the product $\boldsymbol{U}_{r}^{T} \boldsymbol{U}_{r}$ results in a diagonal matrix. So we have found a null space of $\boldsymbol{Q}\left(\rho_{i}\right)$ with the $p$ last columns of $\boldsymbol{U}_{r}$ as a basis, i. e., $\boldsymbol{S}\left(\rho_{i}\right)$ consists of the last $p$ columns of $\boldsymbol{U}_{r}$. This method has the advantage that the existing poles of the open-loop system or values near to them may still be chosen as eigenvalues for the observer, since these values leads to a stable observer.

Now we need to find $\boldsymbol{e}_{i}$ that yields the corresponding eigenvector $\boldsymbol{l}_{i}$ that is closest to the desired value. Since the eigenvector corresponding to a desired eigenvalue must lie in an allowable subspace spanned by the columns of $\boldsymbol{P}\left(\rho_{i}\right)$. This subspace is determined by the plant matrix $\boldsymbol{A}$, the output matrix $\boldsymbol{C}$ and the desired eigenvalue. As normally, the desired eigenvector is not possible, so, we must find an allowable eigenvector in the subspace, which is closest to the desired vector.

As only a few components of the desired vector $\boldsymbol{l}_{i}^{d}$ are usually specified, remain components may be chosen arbitrarily. Ordering the rows through a transformation matrix $\boldsymbol{R}$ we get:

$$
\boldsymbol{R} l_{i}^{d}=\left(\begin{array}{c}
l_{i}{ }^{C} \\
x \\
\vdots \\
x
\end{array}\right)=\boldsymbol{R} \boldsymbol{P}\left(\rho_{i}\right) \boldsymbol{e}_{i}=\left[\begin{array}{c}
\boldsymbol{R}_{1} \\
\boldsymbol{R}_{2}
\end{array}\right] \boldsymbol{e}_{i} ; i=1, \ldots, p
$$

where $\boldsymbol{l}_{i}^{C}$ are the specified components due the constraint and $x$ are free componentes.

Lemma 5: According to [9], the least square solution to an inconsistent system $\boldsymbol{A} \boldsymbol{x}=\boldsymbol{b}$ of $n$ equations and $p$ unknowns $(n>p)$ satisfies $\boldsymbol{A}^{T} \boldsymbol{A} \overline{\boldsymbol{x}}=\boldsymbol{A}^{T} \boldsymbol{b}$. If the columns of $\boldsymbol{A}$ are linearly independent, then $\boldsymbol{A}^{T} \boldsymbol{A}$ is invertible and $\overline{\boldsymbol{x}}=\left(\boldsymbol{A}^{T} \boldsymbol{A}\right)^{-1} \boldsymbol{A}^{T} \boldsymbol{b}$. The projection of vector $\boldsymbol{b}$ onto the column space of $\boldsymbol{A}$ is therefore $\boldsymbol{p}=\boldsymbol{A} \overline{\boldsymbol{x}}=\boldsymbol{A}\left(\boldsymbol{A}^{T} \boldsymbol{A}\right)^{-1} \boldsymbol{A}^{T} \boldsymbol{b}$. The proof can be found in [9]. get:

According to [1] and applying Lemma 5 to eq (23) we

$$
\boldsymbol{e}_{i}=\left[\boldsymbol{R}_{1}^{T} \boldsymbol{R}_{1}\right]^{-1} \boldsymbol{R}_{1}{ }^{T} \boldsymbol{l}_{i}^{C}
$$

We can verify if one vector lies in the subspace through its projection on this subspace. Applying Lemma 5, if $p=b$ then the vector lies in the subspace otherwise it doesn't lie.

The remain vectors $(i=p+1, \ldots, n)$ are calculated in the same way, but remembering that there are no constraints for them.

Now, considering that $\boldsymbol{L}_{\boldsymbol{l}}=\left\{\boldsymbol{l}_{i}\right\}_{i=1 \ldots n}$ is a non singular matrix we can determine the observer gain $\boldsymbol{L}$ from eq. (24) as: 


$$
\boldsymbol{L}^{T} \boldsymbol{L}_{\boldsymbol{l}}=\boldsymbol{Z} \Rightarrow \boldsymbol{L}=\left(\boldsymbol{Z} \boldsymbol{L}_{l}^{-1}\right)^{\mathrm{T}}
$$

The procedure to find $\boldsymbol{W}$ and $\boldsymbol{L}$ can be summarized as

1. Compute $\boldsymbol{W}$ so that $\boldsymbol{W C E}=0$. This determines the left eigenvectors of the observer dynamics in dual control form, according to Lemma 2.

2. Define the desired behavior of the residuals, i. e., where the observer's poles should be placed.

3. Find the corresponding $\boldsymbol{L}$ that generates the desired eigenstructure, i. e., the $\boldsymbol{L}$ that generates the matrix $\boldsymbol{A}_{o}$ with the desired eigenvalues and eigenvectors.

Note: the right eigenvector of the observer dynamics in dual control form is the observer dynamics left eigenvector.

Eigenvector Direction Design - This approach can be done through the Beard and Jones methodology. Eqs. (6) and (7) are used considering $\boldsymbol{f}_{c}=\boldsymbol{f}_{s}=\boldsymbol{d}=0$ and that $\boldsymbol{f}_{a i}$ is one right eigenvector $\boldsymbol{v}_{i}$ of $\mathbf{A}_{\mathbf{0}}$, which represents a single fault in one actuator, so we get:

$$
\begin{aligned}
\dot{e} & =A_{o} e+v_{i} n \\
r & =C e
\end{aligned}
$$

Solving this equation in frequency domain we get:

$$
\begin{aligned}
& \boldsymbol{E}(s)=\left(s \boldsymbol{I}-\boldsymbol{A}_{o}\right)^{-1} \boldsymbol{v}_{i} N(s) \\
& \boldsymbol{R}(s)=\boldsymbol{C}\left(s \boldsymbol{I}-\boldsymbol{A}_{o}\right)^{-1} \boldsymbol{v}_{i} N(s)
\end{aligned}
$$

From Lemma 3 we have:

$$
\begin{aligned}
\boldsymbol{E}(s) & =\left(\frac{\boldsymbol{I}}{s}+\frac{\boldsymbol{A}}{s^{2}}+\cdots+\frac{\boldsymbol{A}^{r}}{s^{r+1}}+\cdots\right) \boldsymbol{v}_{i} N(s) \\
& =\left(\frac{\boldsymbol{v}_{i}}{s}+\frac{\boldsymbol{A} \boldsymbol{v}_{i}}{s^{2}}+\cdots+\frac{\boldsymbol{A}^{r} \boldsymbol{v}_{i}}{s^{r+1}}+\cdots\right) N(s)
\end{aligned}
$$

Applying Lemma 2 to eq. (33)

$$
\boldsymbol{E}(s)=\left(\frac{1}{s}+\frac{\rho_{i}}{s^{2}}+\cdots+\frac{\rho_{i}^{r}}{s^{r+1}}+\cdots\right) \boldsymbol{v}_{i} \boldsymbol{N}(s)
$$

Returning to time domain we get:

$$
\begin{aligned}
& \boldsymbol{e}=e^{\rho_{i}} \boldsymbol{v}_{i} n \\
& \boldsymbol{r}=\boldsymbol{C} e^{\rho_{i}} \boldsymbol{v}_{i} n
\end{aligned}
$$

Eqs. (34) and (35) show that, for the case where the fault $\boldsymbol{f}_{a i}=\boldsymbol{v}_{i}$, the residual vector has a fixed direction $\boldsymbol{C} \boldsymbol{v}_{i}$ independently of $n(t)$ values. So this direction is associated to the fault in the $i^{\text {th }}$ actuator.

Now it is necessary to calculate the $\boldsymbol{L}$ matrix to get the matrix $\boldsymbol{A}_{o}$ with the desired eigenstructure. From Lemma 2 we have:

$$
\boldsymbol{A}_{o} \boldsymbol{v}_{i}=(\boldsymbol{A}-\boldsymbol{L C}) \boldsymbol{v}_{i}=\rho_{i} \boldsymbol{v}_{i}
$$

so

$$
\left(\rho_{i} \boldsymbol{I}-\boldsymbol{A}\right) \boldsymbol{v}_{i}+\boldsymbol{L} \boldsymbol{C} \boldsymbol{v}_{i}=0
$$

Solving eq. (36) we get:

$$
\boldsymbol{L C} \boldsymbol{v}_{i}=\left(\boldsymbol{A}-\rho_{i} \boldsymbol{I}\right) \boldsymbol{v}_{i}
$$

and finally,

$$
\boldsymbol{L}=\left[\boldsymbol{L} \boldsymbol{C} \boldsymbol{v}_{1}, \boldsymbol{L} \boldsymbol{C} \boldsymbol{v}_{2}, \ldots, \boldsymbol{L} \boldsymbol{C} \boldsymbol{v}_{r}\right](\boldsymbol{C F})^{-1}
$$

Modified Detection Filter for Sensor Faults - In this technique, we design the eigenvector of the observer dynamics to have the same directions of the fault that we need to detect. This method is based on the Detection Filter developed by Beard (1971) and Jones (1973) for detecting and identifying faults in Linear Time Invariant Control Systems; and later modified by Mello (1991), and extended and consolidated by Souza and Mello (1997).

The main idea of the modified detection filter is to do a similarity transformation of the mathematical model of the system and make a projection of the residue to the plane orthogonal to the main axis of the sensor fault. Applying the nonsingular similarity transformation matrix $\boldsymbol{T}$ to the model: $\boldsymbol{x}_{t}=\boldsymbol{T} \boldsymbol{x}, \boldsymbol{A}_{t}=\boldsymbol{T A T}, \boldsymbol{B}_{t}=\boldsymbol{T B}, \boldsymbol{C}_{t}=\boldsymbol{C T}$; we get:

$$
\begin{aligned}
& \boldsymbol{A}_{t}=\left[\begin{array}{cc}
\boldsymbol{A}_{t 11} & \boldsymbol{A}_{t 12} \\
0 & \boldsymbol{A}_{t 22}
\end{array}\right] \\
& \boldsymbol{C}_{t}=\left[\begin{array}{ll}
\boldsymbol{I}_{p} & 0
\end{array}\right]
\end{aligned}
$$

where $\boldsymbol{A}_{t 11} \in \mathfrak{R}^{p \times p}, \quad \boldsymbol{A}_{t 12} \in \mathfrak{R}^{p \times(n-p)}, \quad \boldsymbol{A}_{t 21}=0 \in \mathfrak{R}^{(n-p) \times p}$, $\boldsymbol{A}_{t 22} \in \boldsymbol{R}^{(n-p) \times(n-p)} \quad \boldsymbol{C}_{t 11}=\boldsymbol{I}_{p} \in \mathfrak{R}^{p \times p}$ and $\boldsymbol{C}_{t 12}=\underline{\mathbf{0}}$ Lemma 6_-_The canonical form according to [10] can be obtained by:

1. $\boldsymbol{T}$ has the form $\boldsymbol{T}=\left[\begin{array}{ll}\boldsymbol{C} & \boldsymbol{W}\end{array}\right]^{T}$ and $\boldsymbol{T}^{1}=\left[\begin{array}{ll}\boldsymbol{P} & \boldsymbol{Q}\end{array}\right]$, which define $\boldsymbol{P}$ and $\boldsymbol{Q}$.

2. $\boldsymbol{W}$ is defined by $(n-p)$ left eigenvectors of $\boldsymbol{A}$ that complete a set of $\mathrm{n}$ linearly independent rows in $\boldsymbol{T}$.

The proof can be found in [10].

Lemma $7-\boldsymbol{A}_{t 22}$ is diagonal submatrix and it is formed by the $(n-p)$ eigenvalues $\rho_{j}$ correspondent to the $(n-p)$ row eigenvectors $w_{j}$ included in $\boldsymbol{W}$. The proof can be found in [10].

Theorem 3 - Let the $p$ sensor faults distribution matrix be represented by:

$$
\boldsymbol{F}=\left[\begin{array}{llll}
\boldsymbol{f}_{1} & \boldsymbol{f}_{2} & \cdots & \boldsymbol{f}_{p}
\end{array}\right]=\left[\begin{array}{c}
\boldsymbol{I}_{p} \\
\boldsymbol{0}
\end{array}\right]=\left[\begin{array}{cccc}
\boldsymbol{v}_{t_{1}} & \boldsymbol{v}_{t_{2}} & \cdots & \boldsymbol{v}_{t_{p}} \\
\boldsymbol{0} & \boldsymbol{0} & \cdots & \boldsymbol{0}
\end{array}\right]
$$

The matrix $\boldsymbol{L}_{t}$, called observer gain matrix, is designed considering that $\boldsymbol{f}_{i}$ is a right eigenvector of $\boldsymbol{A}_{o t}=\left[\boldsymbol{A}_{t}-\boldsymbol{L}_{t} \boldsymbol{C}_{t}\right]$, 
corresponding to its eigenvalues $\rho_{i}$. The eigenvalues shall be chosen to keep the observer filter stable. If $\boldsymbol{e}(0)=\mathbf{0}$ and if one unknown single fail $\boldsymbol{f}_{i}$ in the $i^{\text {th }}$ sensor starts at $t_{0}, \boldsymbol{e}(\mathrm{t})$ will have a corresponding and normally distinct direction given by: $\boldsymbol{L}_{t i}=\rho_{i} f_{i}+\boldsymbol{F} \boldsymbol{A}_{t l 1}{ }^{i}$, where $\boldsymbol{A}_{t l 1}{ }^{i}$ is the $i^{t h}$ column of $\boldsymbol{A}_{t l 1} \boldsymbol{r}(t)$ will stay in a plane spanned by $\boldsymbol{C} \boldsymbol{L}_{t i}$ and $\boldsymbol{v}_{t i}$. If $\boldsymbol{e}(0) \neq \mathbf{0}$ then $\boldsymbol{r}(t)$ will converge asymptotically to a plane spanned by $\boldsymbol{C} \boldsymbol{L}_{t i}$ and $\boldsymbol{v}_{t i}$. The proof can be find in [10].

Additional Considerations - The final step of the procedure is to compare the direction of the residue vector against the fault directions patterns with the objective of detect and isolate the fault.

A general IFD scheme based on analytical redundancy, basing in [4], can be illustrated as in Figure 4. A block with measurements and control signals as input and a fault decision as output represents it.

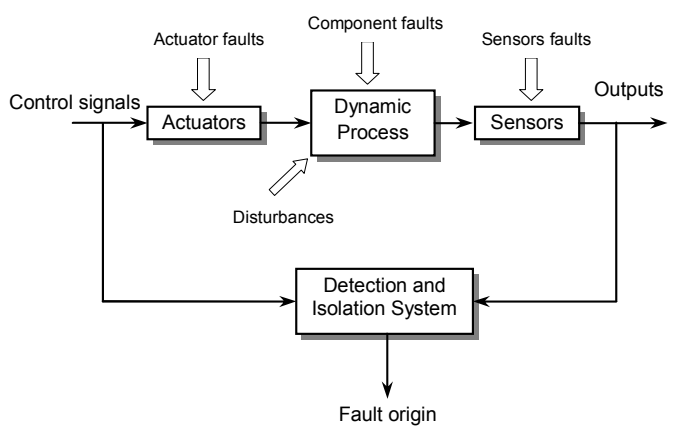

Figure 4 - Structure of an IFD system.

Once the fault is detected and isolated, the control law can be reconfigured, i. e., the fault signal can be changed for another redundant signal (in a Instrument Fault Detection, Isolation and Reconfiguration System - IFDIR system). In case where there is more than one redundant signal, it should be selected that which presents better information quality for the desired signal.

\section{MODEL OF STUDY}

BIBLIOGRAPHICAL REVISION - Following there are some revisions of good references for a SVL such as [5], [6],[13], [16]. The detail project for a fault detection and isolation system using product of two error functions can be found In [5], [6] and [13]. The structure of the control system used in the Brazilian VLS, with focus in description of the algorithms used in its control system, can be found in [16], where there are indications of several references for this vehicle. Several methods for fault detection and isolation in dynamic systems can be found in [1], [2], [3], [4], [11], [12], [14]. The mathematical model to describe the longitudinal motion of a satellite launcher vehicle can be obtained in [15]. The method used for the observers' design using eigenstructure can be found in [1], [4] and [10]. The detection and isolation of actuators and sensors faults, using the modified fault detection filter, can be found in [13].

DESCRIPTION - The mathematical model used for this study is a simplified model of longitudinal motion of the satellite launcher vehicle (SLV) describe in [13]. As these types of vehicles are unstable, the fault in a sensor can be catastrophic if the control system doesn't have any redundancy degree, physical or analytical. The detailed description of the basic model used can be found in [13] and complemented in [5]. To this model we added the row $\dot{z}=z$ to the system dynamic matrix and the measure $y_{w}=w+z$ to the measurement matrix keeping the system completely observable and ease apply the eigenstructure method. With the above consideration the following model was used to the system longitudinal model:

$$
\begin{aligned}
& {\left[\begin{array}{c}
\dot{w} \\
\dot{q} \\
\dot{\theta} \\
\dot{z}
\end{array}\right]=\left[\begin{array}{cccc}
Z_{w} & Z_{q}+U_{0} & -g & 0 \\
M_{w} & M_{q} & 0 & 0 \\
0 & 1 & 0 & 0 \\
0 & 0 & 0 & 1
\end{array}\right] *\left[\begin{array}{c}
w \\
q \\
\theta \\
z
\end{array}\right]+\left[\begin{array}{c}
Z_{\beta z} \\
M_{\beta z} \\
0 \\
0
\end{array}\right] * \beta_{z}} \\
& \boldsymbol{y}=\left[\begin{array}{llll}
1 & 0 & 0 & 1 \\
0 & 1 & 0 & 0 \\
0 & 0 & 1 & 0
\end{array}\right] \boldsymbol{x}+\boldsymbol{D} \boldsymbol{u}
\end{aligned}
$$

where $\boldsymbol{D}=\mathbf{0}, w$ denotes the linear velocity along the $z$ axis in the vehicle system, denominated normal velocity; $q$ is the pitch-rate velocity, that is, the angular velocity around the $y$ axis in the vehicle system; $\theta$ is the pitch attitude, that is, the angle of the attitude around the $y$ axis in the vehicle system; $z$ is the state added for illustration of the method; $\beta_{Z}$ is the deflection angle of the actuator around the $y$ axis in the vehicle system; $Z_{w}, Z_{q}, M_{w}, M_{q}, Z_{\beta z}$ e $M_{\beta z}$ are the aerodynamics derivatives of the satellites launcher vehicle, obtained in wind tunnel tests; $U_{0}$ is the module of the linear velocity of the vehicle, and $g$ is the local gravity acceleration.

Table 1 - Parameters used in the model and gains of the control law.

\begin{tabular}{|c|c|}
\hline Parameter & Value \\
\hline$Z_{w}\left[\mathrm{~s}^{-2}\right]$ & $-0,0968$ \\
\hline$Z_{q}\left[\mathrm{~s}^{-2}\right]$ & 0,1631 \\
\hline$M_{w}\left[\mathrm{~m}^{-1} \mathrm{~s}^{-1}\right]$ & 0,0096 \\
\hline$M_{q}\left[\mathrm{~s}^{-1}\right]$ & 0,0568 \\
\hline$Z_{\beta z}\left[\mathrm{~m} \mathrm{~s}^{-2}\right]$ & 19,3761 \\
\hline$M_{\beta z}\left[\mathrm{~s}^{-2}\right]$ & 7,2769 \\
\hline$U_{0}\left[\mathrm{~m} \mathrm{~s}^{-1}\right]$ & 544,46 \\
\hline$g\left[\mathrm{~m} \mathrm{~s}^{-2}\right]$ & 9,7886 \\
\hline
\end{tabular}

Design 1-Applying the Robust Residual Generator methodology, where we need a robust residual generator insensitive to actuator fault with the following eigenvalues $\boldsymbol{\rho}=[-7 ;-5 ;-4 ;-3]$, to keep the observer dynamics stable and 
faster than system dynamic. The plant eigenvalues are:

\section{$\lambda=[-2.3160 ; 2.2587 ; 0.017958 ; 1.0000]$}

The pair $(A, C)$ is completely observable, so from eq. (16), we get:

$$
\left\{\begin{array}{l}
19.376 w_{11}+7.2769 w_{12}=0 \\
19.376 w_{21}+7.2769 w_{22}=0 \\
19.376 w_{31}+7.2769 w_{32}=0
\end{array}\right.
$$

From Theorem 2 we get:

$$
\left\{\begin{array}{l}
l_{11}-l_{41}=0 \\
l_{12}-l_{42}=0 \\
l_{13}-l_{43}=0
\end{array}\right.
$$

So the similarity transformation matrix is:

$$
\boldsymbol{T}=\left[\begin{array}{cccc}
19.376 & 7.2769 & 0 & 0 \\
1 & 0 & 0 & -1 \\
0 & 0 & 1 & 0 \\
0 & 0 & 0 & 1
\end{array}\right]
$$

According to [4], the desired eigenvectors $\boldsymbol{l}_{i}^{\mathrm{T}}$ $(i=1, \ldots, 3)$ were chosen as the row in $\boldsymbol{W C}$. The $\boldsymbol{l}_{4}$ vector was chosen from one of the columns of $\boldsymbol{P}(-3)$. So we got:

$$
\boldsymbol{L}_{l}{ }^{\mathrm{T}}=\left[\begin{array}{cccc}
1.133 & -3.0168 & 0 & 0.97406 \\
0 & 0 & 1 & 0 \\
1.1898 & -3.1679 & 1 & 0.9227 \\
0.0055904 & -0.99979 & 0.017024 & 0.001658
\end{array}\right]
$$

Note that the constraints from eq. (41) are only approximately fulfilled, as mentioned in [1].

From eq. (28) we got the observer gain:

$$
\boldsymbol{L}=\left[\begin{array}{ccc}
-23.979 & 612.2 & -34,78 \\
-0.08161 & 3.2997 & -0.054352 \\
0 & 1 & 5 \\
35.639 & -94.895 & 30.955
\end{array}\right]
$$

Finally we got the following observer dynamic $\boldsymbol{A}_{o}$ and $W$ matrix:

$$
\boldsymbol{A}_{o}=\boldsymbol{A}-\boldsymbol{L C}=\left[\begin{array}{cccc}
23.882 & -71.578 & 24.992 & 23.979 \\
0.09121 & -3.2429 & 0.054352 & 0.08161 \\
0 & 0 & -5 & 0 \\
-35.639 & 94.895 & -30.955 & -34.659
\end{array}\right]
$$

$$
\boldsymbol{W}=\left[\begin{array}{ccc}
1.133 & -3.0168 & 0 \\
0 & 0 & 1 \\
1.1898 & -3.1679 & 1
\end{array}\right]
$$

Desing 2 - Applying the Modified Detection Filter methodology, where we need that the eigenvector of the observer dynamics has the same direction of the fault that we want to detect. The plant eigenvalues and left eigenvectors are:

$$
\lambda=[-2.3160 ; 2.2587 ; 0.017958 ; 1.0000]
$$

$$
\boldsymbol{V}_{l}=\left[\begin{array}{cccc}
-0.0043245 & 1 & -0.01872 & 0 \\
0.0040755 & 1 & -0.017662 & 0 \\
-0.0018346 & -0.02193 & 1 & 0 \\
0 & 0 & 0 & 1
\end{array}\right]
$$

The pair $(\boldsymbol{A}, \boldsymbol{C})$ is completely observable, so we need to do one similarity transformation. To compound the matrix $\boldsymbol{T}$ we will use the left eigenvector $\boldsymbol{W}=\left[\begin{array}{llll}-0.0043245 & 1 & -0.018272 & 0\end{array}\right]$ from the matrix $\boldsymbol{V}_{l}$, corresponding to eigenvalue $\lambda_{1}=-2.316$. So we get:

$$
\begin{aligned}
& \boldsymbol{T}=\left[\begin{array}{cccc}
1 & 0 & 0 & 1 \\
0 & 1 & 0 & 0 \\
0 & 0 & 1 & 0 \\
-0.0043245 & 1 & -0.018272 & 0
\end{array}\right] \\
& \boldsymbol{A}_{t}=\boldsymbol{T A T}^{-1}=\left[\begin{array}{cccc}
1 & 291.0 & -5.1544 & 253.62 \\
0 & 2.2767 & -0.040562 & -2.2199 \\
0 & 1 & 0 & 0 \\
0 & 0 & 0 & -2.3167
\end{array}\right] \\
& \boldsymbol{B}_{t}=\boldsymbol{T} \boldsymbol{B}=\left[\begin{array}{c}
19.376 \\
7.2769 \\
0 \\
7.1931
\end{array}\right] \quad \boldsymbol{C}_{t}=\boldsymbol{C T}^{-1}=\left[\begin{array}{cccc}
1 & 0 & 0 & 0 \\
0 & 1 & 0 & 0 \\
0 & 0 & 1 & 0
\end{array}\right] \\
& \text { Let } \boldsymbol{F}_{t}=\left[\begin{array}{lll}
1 & 0 & 0 \\
0 & 1 & 0 \\
0 & 0 & 1 \\
0 & 0 & 0
\end{array}\right] \text { be the set of } p \text { sensor faults to be }
\end{aligned}
$$
used in the design of the Modified Detection Filter. Choosing the Observer eigenvalues $\rho_{1}=\rho_{2}=\rho_{3}=\rho$ and applying Theorem 3 we get:

$$
\boldsymbol{l}_{i}=\left[\begin{array}{c}
-\rho \boldsymbol{v}_{i}+\boldsymbol{A}_{t 11}^{i} \\
0
\end{array}\right] \text { where } \boldsymbol{A}_{t 11}^{i} \text { is the } i^{t h} \text { column of the }
$$

matrix $\boldsymbol{A}_{t 11}$. As $\rho_{1}=\rho_{2}=\rho_{3}=\rho$ we can use: 
$\boldsymbol{L}=\left[\begin{array}{c}\boldsymbol{A}_{t 11}-\rho \boldsymbol{I}_{p} \\ 0\end{array}\right]=\left[\begin{array}{ccc}1-\rho & 291.0 & -5.1544 \\ 0 & 2.2767-\rho & -0.040562 \\ 0 & 1 & -\rho \\ 0 & 0 & 0\end{array}\right]$

So we get:

$$
\boldsymbol{A}_{o t}=\left[\begin{array}{cc}
\rho \boldsymbol{I}_{p} & \boldsymbol{A}_{t 12} \\
0 & \boldsymbol{A}_{t 22}
\end{array}\right]=\left[\begin{array}{cccc}
\rho & 0 & 0 & 253.62 \\
0 & \rho & 0 & -2.2199 \\
0 & 0 & \rho & 0 \\
0 & 0 & 0 & -2.3167
\end{array}\right]
$$

Calculating the residue dynamics in frequency domain we get:

$\left[s \boldsymbol{I}-\boldsymbol{A}_{o t}\right]^{-1}=\left[\begin{array}{cccc}\frac{1}{s-\rho} & 0 & 0 & \frac{253.62}{(s+2.3167)(s-\rho)} \\ 0 & \frac{1}{s-\rho} & 0 & \frac{2.2199}{(s+2.3167)(s-\rho)} \\ 0 & 0 & \frac{1}{s-\rho} & 0 \\ 0 & 0 & 0 & \frac{1}{(s+2.3167)}\end{array}\right]$

Now determining the vectors direction for single fault in the sensors using eq. (7) in frequency domain and considering $f_{a}=f_{c}=d=\boldsymbol{0}$ we get:

$$
\boldsymbol{R}_{i}(s)=\frac{-N(s)}{s-\rho} \boldsymbol{C}_{t} \boldsymbol{L}_{i}+N(s) \boldsymbol{v}_{i}
$$

The vector direction for fault at sensor 1 is:

$$
\begin{gathered}
\boldsymbol{R}_{1}=\frac{-N(s)}{s-\rho}\left[\begin{array}{cccc}
1 & 0 & 0 & 0 \\
0 & 1 & 0 & 0 \\
0 & 0 & 1 & 0
\end{array}\right]\left[\begin{array}{c}
1-\rho \\
0 \\
0 \\
0
\end{array}\right]+N(s)\left[\begin{array}{l}
1 \\
0 \\
0
\end{array}\right] \\
\boldsymbol{R}_{1}=\frac{N(s)}{s-\rho}\left[\begin{array}{c}
\rho-1 \\
0 \\
0
\end{array}\right]+N(s)\left[\begin{array}{l}
1 \\
0 \\
0
\end{array}\right]
\end{gathered}
$$

The vector direction for fault at sensor 2 is:

$$
\boldsymbol{R}_{2}=\frac{N(s)}{s-\rho}\left[\begin{array}{c}
-291.0 \\
-2.2767+\rho \\
1
\end{array}\right]+N(s)\left[\begin{array}{l}
0 \\
1 \\
0
\end{array}\right]
$$

The vector direction for fault at sensor 3 is:

$$
\boldsymbol{R}_{3}=\frac{N(s)}{s-\rho}\left[\begin{array}{c}
5.1544 \\
0.040562 \\
\rho
\end{array}\right]+N(s)\left[\begin{array}{l}
0 \\
0 \\
1
\end{array}\right]
$$

FAULT CASE STUDY - To see the performance of the IFD system we used the Simulink Simulation
Environment, version 5.0, from MatLab, version 6.5.0. We discarded the fault $f_{c}$ and the disturbance $d$, presented in eq. (4). To facilitate the visualization of the result for a single fault we used the unit step function for the fault functions $f_{a}(t)$ and $f_{s}(t)$. We used the integration method "ODE5 - Dormand-Prince" from MATLAB for simulation.

For design 1 we considered a step fault in the actuator at $1 \mathrm{~s}$ and a step fault in one sensor at $5 \mathrm{~s}$. In Figure 5 is presented the actuator and the $w$ sensor fault signals applied to the system. In Figure 6 we can see that the system is insensitive to actuator fault.

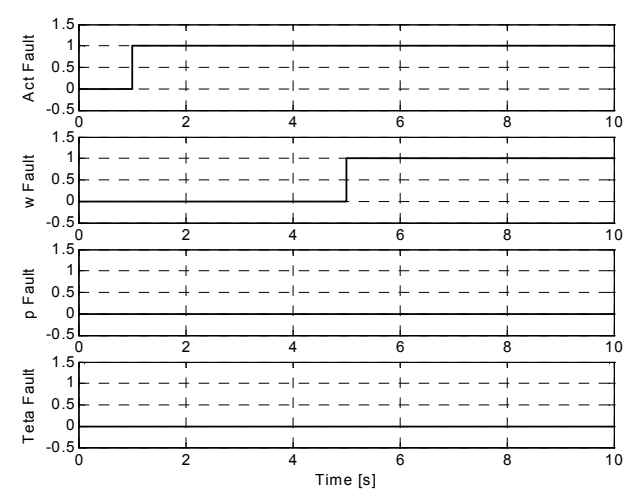

Figure 5 - Fault signals applied to the system.
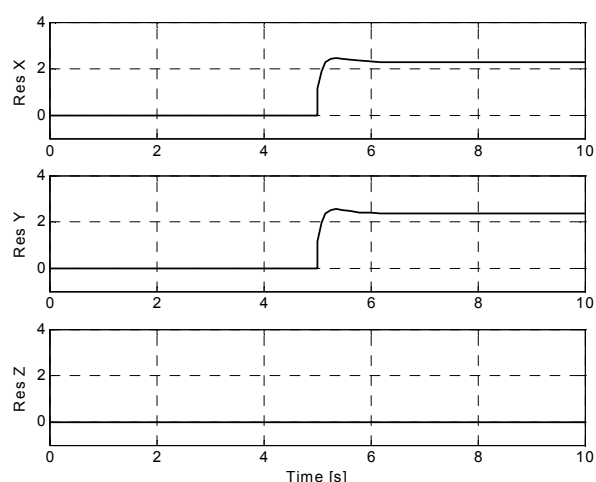

Figure 6 - Residuals due the faults applied.

In Figure 7 we have the residue vector for the actuator and $w$ sensor faults. The solid lines represent the amplitude of the residue. In this case the residue vector has only one direction and lies on the plane $\mathrm{XY}$.

In Figure 8 we have the residue vector for the actuator and $p$ sensor faults. For a graphical representation we used the axis $X$ to represent the component Res $X$ from the residual vector, axis $\mathrm{Y}$ for Res $\mathrm{Y}$ and axis $\mathrm{Z}$ for Res $\mathrm{Z}$. The solid lines represents the residual during the simulation, where one extremity of the residue pass by the origin and 
the other has a interpolation line for visualization of the vector variation. In this case the residual lies on the plane approximately perpendicular to the plane XY. Note that Z values are very small in comparison with $\mathrm{X}$ and $\mathrm{Y}$ values. The dots are for the vector amplitude projection to the plane $\mathrm{XY}$.

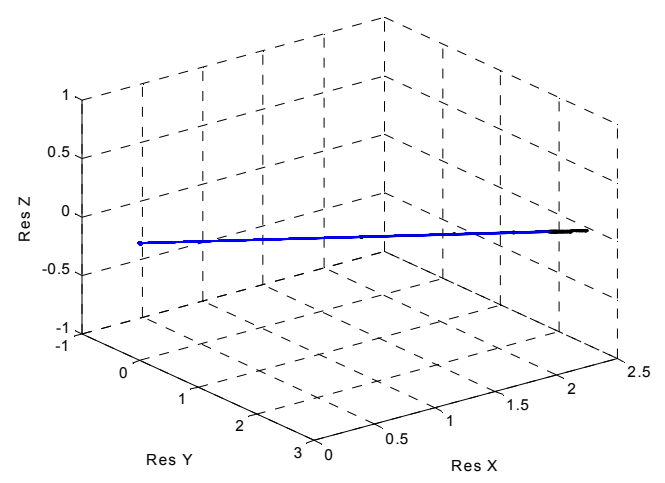

Figure 7 - Residue vector for actuator and $w$ sensor faults.

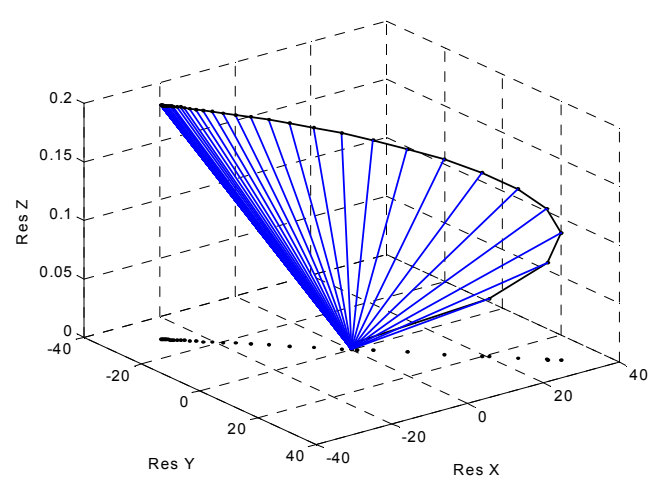

Figure 8 - Residue vector for actuator and $p$ sensor faults.

In Figure 9 we have the residue vector for the actuator and $\theta$ sensor faults. Note now that $\mathrm{Z}$ values have approximately the same magnitude as $\mathrm{X}$ and $\mathrm{Y}$ values. The dots show that the residuals do not lie on a plane.

For design 2 we considered only a step fault in one of the sensors for each simulation at $1 \mathrm{~s}$. The solid lines represents the residual during the simulation, where one extremity of the residue pass by the origin and the other has a interpolation line for visualization of the vector variation. The dots are for its projection to the plane perpendicular to the main residual axis. For a graphical representation we used the axis Res $\mathrm{X}$ as a main axis for $w$ sensor fault, axis
Res $\mathrm{Y}$ for $p$ sensor fault and axis Res Z for $\theta$ sensor fault.

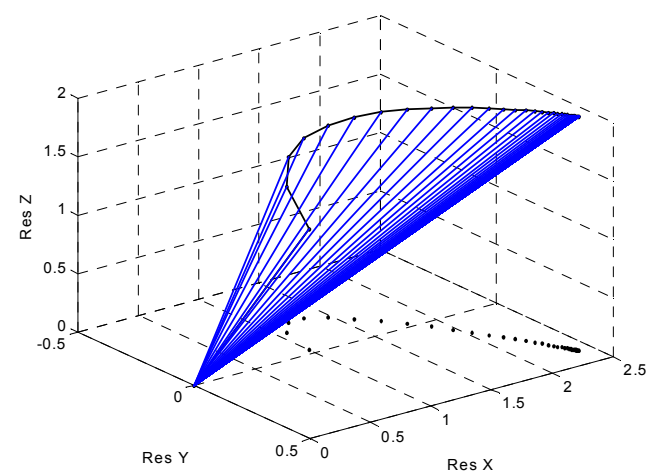

Figure 9 - Residue vector for actuator and $\theta$ sensor faults.

In Figure 10 we have the residue for a fault in the $w$ sensor. Note that in this case the residue vector is parallel to the Res $\mathrm{X}$ axis, i. e., it is a case where the residual lies on line as mentioned before. So in this case its projection on the plane perpendicular to this axis is null and the residual must be tested with the $\mathrm{X}$ axis.

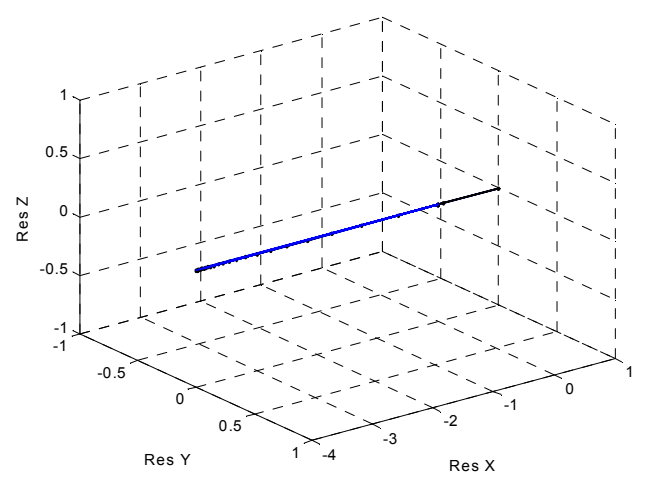

Figure 10 - IFD System response for a step fault in $w$.

In Figure 11 we have residue for a fault in the $p$ sensor. The residue vector forms a plane that contains the Y-axis. Note in this case the difference of magnitude between the $\mathrm{X}$-axis and the others axes. It can be difficult to determine the angle in noise presence to isolate a fault in the $w$ sensor.

In Figure 12 we have residue for a fault in the $\theta$ sensor. The residue vector also form a plane but now contain the $\mathrm{Z}$ axis. 


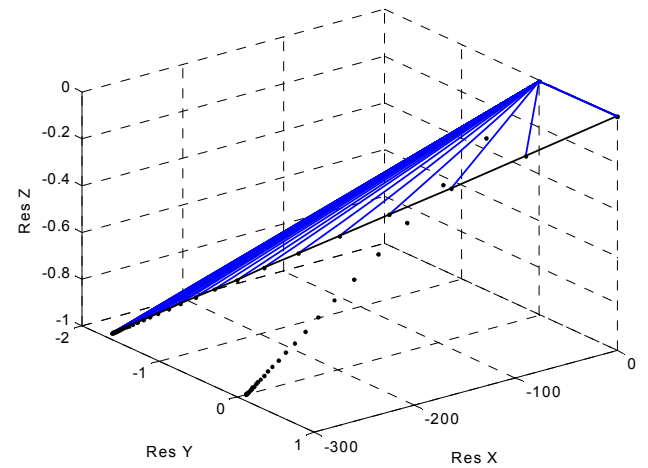

Figure 11 - IFD System response for a step fault in $p$.

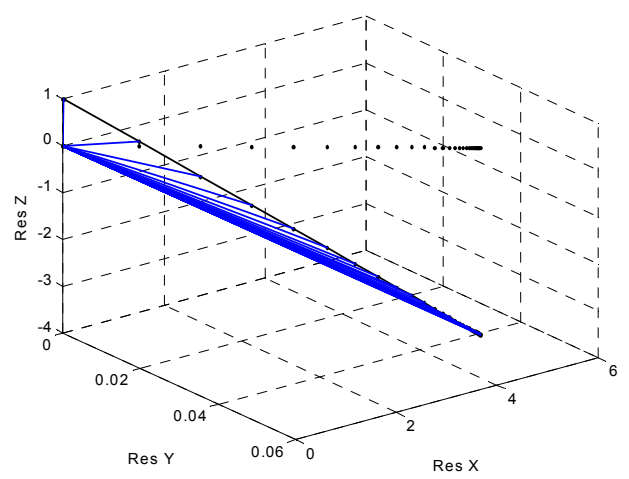

Figure 12 - IFD System response for a step fault in $\theta$.

\section{COMMENTS AND CONCLUSIONS}

It should be considered in the observers design that they should be considered the case that initial conditions the plant are not null and it can cause a false alarm indication for all sensors when the process is initiated.

This method detection and isolating the fault based on fault direction vectors presents the advantage of being able to detect fault since when there is parameters variation of the plant or in presence of noise in the measure. For the Robust Residual Generator it has the advantage that the residual can be insensitive to unknowns inputs. In case of the method of the Modified Detection Filter it has the advantage that it has strong fault detectability [4] to indicate sensor fault but it is not applicable for actuators, components or disturbance to the plant.

For the methodology presented it was noticed that the IFD system has a good performance to detect and isolate abrupt faults type but its performance decrease in cases of intermittent fault due to the time response of the observer designed.

\section{BIBLIOGRAPHICAL REFERENCES}

[ 1] - Patton, R.; Frank, P. and Clark, R., "Fault Diagnosis in Dynamics Systems: Theory and Applications". Prentice Hall Int. Series in Systems and Control Engineering, Englewood Cliffs, NJ, 1989.

[ 2]-Gertler, Janos J., "Survey of Model-Based Failure Detection and Isolation in Complex Plants". IEEE Control Systems Magazine, Vol. 8, no 6, pp. 3-11, December 1988.

[3]-Chow, E. Y. and Willsky, A. S., "Analytical Redundancy and the Design of Robust Failure Detection Systems". IEEE Trans. Automatic Control, Vol. AC-29, $\mathrm{n}^{\mathrm{o}}$ 7, pp. 603-614, July 1984.

[ 4]-Frisk, E., "Model-based Fault Diagnosis Applied to a SI-Engine". site www.vehicular.isy.liu.if, Linköping University, Linköping, Sweden, 1996.

[ 5] - Teixeira, A. J., Souza, M. L. O. and Oliva, A. P., "Techniques For Fault Diagnosis In Aerospace And Automotive Systems". In SAE BRASIL 2003 Congress, site www.saebrasil.org.br, nov 2003

[ 6] - Teixeira, A. J., Souza, M. L. O. and Oliva, A. P., "An Algorithm for Instrument Fault Detection in Inertial Sensors of a Satellite Launcher Vehicle Control System". In COBEM 2003 Congress, 2003.

[ 7] - Chen, C. T., "Introduction to Linear Systems Theory". Holt, Rinehart and Winston Co., New York, 1984.

[ 8] - Moore, B. C., "On the Flexibility Offered by State Feedback in Multivariable Systems Beyond CloseLoop EigenvalueAssignment". IEEE Trans. Automatic Control, Vol. AC-21, 689-692, 1976.

[ 9]-Strang, G., "Linear Algebra and Its Applications". Harcourt Brace Jovanovich College Publishers, 1988.

[10] - Mello, A. F. e Souza M. L. O., "A Modified Detection Filter for Sensor Faults". AAS/AIAA Space Flight Mechanics Meeting, Huntsville, Al., USA, 10-12, Paper AAS97-196, February 1997.

[11]-Patton R. J., "Robust Model-Based Fault Diagnostic State of the Art". In IFAC Fault Detection, Supervision and Safety for Technical Processes, pp. 1-24, Espoo, Finland, 1994. 
[12] - Hsu, P. L., Lin K. L. and Shen, L. C., "Diagnosis of Multiple Sensor and Actuator Failures in Automotive Engines". IEEE transactions on vehicular technology, 44(4):779-789, November 1995.

[13] - Oliva, Álvaro P., "Sensor Fault Detection and Analytical Redundancy Satellite Launcher Flight Control System”. SBA Controle \& Automação, Vol. 9, nº . 3, pp. 156-164, 1998

[14] - Willsky, The. S., "The Survey of Design Methods for Failure Detection in Dynamics Systems". Automatica, Vol. 12, nº 6, pp. 601-611, November 1976.

[15]-McLean, D., "Automatic Flight Control Systems”. Englewood Cliffs. NJ, Prentice Hall Inc, 1990.

[16] - Leite Filho, W. C., "Estrutura do Sistema de Controle do VLS". Controle \& Instrumentação, Ano7, $\mathrm{n}^{\mathrm{o}}$ 72, pp. 71-77, Agosto 2002.

ABOUT THE AUTHOR - Adilson de Jesus Teixeira received the Electronics Engineer degree in 1981 from the University of Parahyba Valley, at S. José dos Campos, São Paulo, Brazil; and his Master of Science degree in Electronics Engineering in 1989 from the Aeronautics Technological Institute in the same city; he is a doctoral candidate in Space Engineering and Technology Course of the National Institute for Space Research-INPE, at the same city; and he is a Development Engineer at the Institute of Aeronautics and Space-IAE of the Aerospace Technological Center-CTA in the same city for more than twenty years.

Email: adilson@iae.cta.br 
